\title{
How mechanical context and feedback jointly determine the use of mechanical variables in length perception by dynamic touch
}

\author{
Rudmer Menger and Rob Withagen \\ University of Groningen, Groningen, The Netherlands
}

\begin{abstract}
Earlier studies have revealed that both mechanical context and feedback determine what mechanical invariant is used to perceive length by dynamic touch. In the present article, the authors examined how these two factors jointly constrain the informational variable that is relied upon. Participants were to judge length while wielding a rod or while holding it stationary. In two experiments, it was tested whether perceptual learning effects in the wielding condition transferred to the holding condition and vice versa. There was an asymmetry in transfer across mechanical conditions: Improvements in the holding context transferred to the wielding condition, but not vice versa. Examining the individuals' exploitation of mechanical variables, we found that, after feedback, participants changed in information usage in both mechanical conditions. For many participants, these changes were not confined to the mechanical context in which the feedback was provided. Indeed, feedback in one mechanical context brought about changes in information usage that often manifested themselves in the other mechanical condition. The authors explore the implications of these findings for research on perceptual learning in dynamic touch and the salience hypothesis.
\end{abstract}

Over the last few decades, numerous studies of perception have aimed to reveal the factors governing the use of informational variables. Following Cutting's (1986, 1991) directed perception approach, several studies demonstrated that what informational variable participants exploit depends on the environmental context in which they have to perform the task. These effects of task ecology on information usage have now been revealed in many paradigms, among which are dynamic touch (van de Langenberg, Kingma, \& Beek, 2006), visually guided interception (e.g., Tresilian, 1999), visual perception of curvature (Cutting \& Millard, 1984), and visual perception of rigid flatness (Cutting, 1986). As an example, and as described below, the information that is relied upon in perceiving length by dynamic touch depends on the mechanical context (rod orientation and exploratory behavior) in which the length has to be judged (see, e.g., van de Langenberg et al., 2006).

Studies of perceptual learning, on the other hand, have demonstrated that participants can change the informational basis of their perception after feedback; that is, in the same environmental context, perceivers have been found to change the information that they exploit as a result of feedback on their performance. The Gibsons (E. J. Gibson, 1963/1991; J. J. Gibson, 1966; J. J. Gibson \& E. J. Gibson, 1955) described this learning process as the education of the perceiver's attention to the right informational variable. Perceivers often start with the detection of variables that relate ambiguously to the environmental property, but move to the exploitation of specifying information after feedback; that is, they converge on the detection of informational variables that relate one-toone to the to-be-perceived property. The recent upsurge in studies of perceptual learning has demonstrated that this process occurs in many tasks: the visual perception of the pulling force of a stick figure (e.g., Michaels \& de Vries, 1998), the visual perception of the relative mass of colliding balls (e.g., Jacobs, Michaels, \& Runeson, 2000; Jacobs, Runeson, \& Michaels, 2001; Runeson \& Andersson, 2007; Runeson, Juslin, \& Olsson, 2000), height and length perception by dynamic touch (Michaels, Arzamarski, Isenhower, \& Jacobs, 2008; Wagman, Shockley, Riley, \& Turvey, 2001; Withagen \& Michaels, 2005; Withagen \& van Wermeskerken, 2009), visually guided braking (Fajen, 2008; Fajen \& Devaney, 2006), and visually guided catching (van Hof, van der Kamp, \& Savelsbergh, 2006).

Recent studies of cultural differences in perception have also revealed a cultural component to the determination of what information is detected (see, e.g., Nisbett, 2003, and Nisbett \& Miyamoto, 2005, for an extensive overview). As an example, Asians and Westerners have been found to exploit different informational variables in the rod-and-frame test - that is, in their judgments of whether a rod appears vertical. And recently, Withagen and van Wermeskerken (2009) argued that there are also organismal factors involved. Inspired by an evolutionary

R.Withagen, r.g.withagen@rug.nl 
analysis of perception and the use of information (Withagen \& Chemero, 2009), they suggested that the observed individual differences in the perceptual learning trajectories reflect between-subjects variation in perceptual learning capacities. That is, participants vary in how well and how quickly they can learn a perceptual task.

Up to this point, studies of variation in information usage have focused almost exclusively on the effects of a single factor in what information is detected. The ongoing discovery of factors that govern information usage, however, suggests that several factors jointly determine what information is exploited. Indeed, as with other variable organismal features (e.g., intelligence and height), the use of information is likely to be a function of the interplay of multiple environmental and organismal factors. To understand how different factors jointly determine what information a perceiver relies on, Withagen and Chemero (2009) recently suggested applying a developmental systems approach to perception (e.g., Ingold, 2000; Oyama, 1985; Thelen \& Smith, 1996). At present, we are far from such a perspective. However, to develop such a view, a shift in focus is required. Instead of studying the factors that seem to influence information usage in isolation, studies should aim at revealing how different factors jointly determine what information is detected. The present article follows this line of thinking.

To examine how several factors jointly constrain information usage, we decided to use the paradigm of length perception by dynamic touch. In this paradigm, participants are to report the length of unseen, handheld rods. We chose this paradigm for two reasons. First, since Solomon and Turvey's (1988) pioneering study of length perception through dynamic touch, several mechanical variables that inform this perception have been revealed (see, e.g., Burton \& Turvey, 1990; Carello, Fitzpatrick, Domaniewicz, Chan, \& Turvey, 1992; Fitzpatrick, Carello, \& Turvey, 1994; Kingma, Beek, \& van Dieën, 2002; Kingma, van de Langenberg, \& Beek, 2004; Turvey, 1996; van de Langenberg et al., 2006). Among these variables are the major principal moment of inertia $\left(I_{1}\right)$, the minor principal moment of inertia $\left(I_{3}\right)$, the first moment of mass distribution $(M)$, and the zeroth moment of mass distribution $(m)$. Second, and related to this, earlier studies have found two factors that determine which mechanical variable(s) participants exploit to perceive length. In a series of experiments, van de Langenberg et al. (see also Kingma et al., 2004) studied how mechanical context (orientation of the rod and exploratory behavior) determines what information is exploited. For example, if participants judged the length while wielding the rod forward, they used $M$ and $I_{1}$. If they held the rod forward, on the other hand, they based their length judgment on $M$. And if they judged length while holding the rod downward, their length estimation was a function of $m$. Van de Langenberg et al. explained their findings with their salience theory. This theory holds that in different mechanical contexts, different mechanical invariants "stand out" in the stimulus flow, and participants will detect the most salient invariant in that flow.

Feedback is another factor that has been found to determine what mechanical invariant is used in perceiving length by dynamic touch. Indeed, several studies have revealed that in the horizontal wielding context, participants can change the mechanical variable they exploit over time (Michaels et al., 2008; Wagman et al., 2001; Withagen \& Michaels, 2005; Withagen \& van Wermeskerken, 2009). Most mechanical variables that the aforementioned studies have revealed relate ambiguously to the length of homogeneous rods. After all, the mechanical invariants $I_{1}, I_{3}, M$, and $m$ are all functions of the rod's radius, length, and material density. This means that rods of the same length but made of different materials can vary in these mechanical invariants, and that rods identical in these invariants can vary in length. Hence, relying on such nonspecifying variables yields inaccuracies in the judgments of length. However, there are several mechanical variables available that specify the length of homogeneous rods (Kingma et al., 2002; Withagen \& Michaels, 2005; Withagen \& van Wermeskerken, 2009). Indeed, any ratio of two moments of mass distribution is specific to the length of thin homogeneous rods. ${ }^{1}$ Consider, for instance, the ratio of the second moment of mass distribution to the first moment of mass distribution,

$$
\frac{I_{1}}{M}=\frac{1 / 3 \cdot m \cdot l^{2}}{1 / 2 \cdot m \cdot l},
$$

where $m$ is mass and $l$ is length. Because the mass cancels, this variable relates one-to-one to rod length. Thus, rods of the same length have the same $I_{1} / M$, irrespective of the material the rods are made of. Earlier studies have revealed that participants can learn to detect a specifying mechanical variable in the wielding condition (Withagen \& Michaels, 2005; Withagen \& van Wermeskerken, 2009; see also Michaels et al., 2008; Wagman et al., 2001); that is, after feedback they change from the exploitation of a nonspecifying mechanical variable to a specifying one.

In the present experiments, we aimed to reveal how mechanical context and feedback jointly determine what mechanical invariant is exploited. As mentioned earlier, what information is relied on is likely to be influenced by multiple factors. Hence, the task at hand is to reveal how different factors jointly determine what information is used. We used a standard pretest-feedback-posttest design and examined the information usage in two mechanical contexts: horizontal wielding and horizontal holding. In Experiment 1, the participants were to hold the rods in the test phases, and wield them in the feedback phases. In Experiment 2, it was the other way around. This design allowed us to examine whether perceptual learning can occur in both contexts and what the effect of perceptual learning is on information usage in the other context. More precisely, if participants receive feedback on their length judgments in one mechanical context, are the possible learning effects apparent in the other mechanical context? Or do participants return to the salient nonspecifying variable that they used in the pretest? It is important to note that, to our knowledge, perceptual learning has not been demonstrated in the horizontal holding context. Because the moments of inertia are detectable only while the rod is wielded and, thus, are not available in this context (see Kingma et al., 2004; van de Langenberg et al., 2006), one 
might wonder whether convergence on a specifying mechanical variable is possible in such a condition. However, in a set of homogeneous rods, there are also in the holding context mechanical variables available that specify length. As mentioned above, any ratio of two moments of mass distribution specifies the length of homogeneous rods. Hence, the ratio of $M$ to $m$ also relates one-to-one to rod length, and these variables are available while a rod is held horizontally. Another related variable was suggested by Hoisington in 1920: When a rod is held horizontally, there is a downward force $(D)$ at the heel of the hand and an upward force $(U)$ at the index finger (Figure 1). The former is felt as an upward kick, the latter as a downward pressure. Hoisington hypothesized that haptic length perception during holding is a function of a force gradient given by the ratio of the downward and upward forces impressed on the tissue of the hand. But if the distance between these two forces is known or constant, this ratio is specific to the length of homogeneous rods. Indeed, as derived by Carello et al. (1992), the relation between the force gradient and the distance between $U$ and $D$, and between $U$ and the center of mass, is as follows:

$$
\frac{D}{U}=\frac{a}{(a+b)}
$$

where $b$ is the distance separating the two forces, and $a$ the distance between $U$ and the center of mass (see Figure 1). Rearranging yields

$$
a=\frac{-b \cdot D}{(D-U)} .
$$

Thus, if $U$ and $D$ are detectable and $b$ is constant or perceivable, $a$ can be known, and because the center of mass is always in the middle of homogeneous rods, this force gradient is related one-to-one to the length of such rods. It is important to note, however, that van den Langenberg et al. (2006) found that $m$ was not implied in perceiving length during horizontal holding. And Carello et al. (1992) found that the force gradient was not a good predictor of length perception in this context. However, these findings might have been the result of using novice perceivers as participants. Indeed, when provided with feedback, perceivers might learn to detect (one of) these variables; so, it is at least theoretically possible that participants learn to perceive the length of homogeneous rods in the holding context.

\section{EXPERIMENT 1}

The aim of Experiment 1 was to test whether learning effects in the wielding context are apparent in the holding context. We used a standard pretest-feedback-posttest design. In the test phases, the participants were to estimate the length of rods while holding them horizontally. In the feedback phases, the participants were to judge the length while wielding the rods. After each judgment in the feedback phase, the participants were informed about the actual length of the handheld rod. To find out whether changes in information usage occurred, we tried to determine what mechanical invariants constrained the length judgments in the different phases of the experiment. In addition, to test whether changing mechanical context or experience with the task had an effect on information usage, we added a control group that judged rod length in each phase but that received no feedback at all. We expected that participants in the experimental group learned to detect one of the specifying variables in the feedback phases. However, because several specifying variables are not available in the holding condition, in the posttest participants might return to the salient nonspecifying variable they started with.

\section{Method}

Participants. Seven men and 5 women volunteered to participate in the experiment and gave informed consent. Eight participants were assigned to the experimental group, 4 participants to the control group. Their ages ranged from 21 to 29 years. All were righthanded.

Materials. Two distinct sets of rods were used. To prevent participants from simply learning to recognize individual rods, one set was used in the feedback blocks, the other set in the test blocks. Each set consisted of 13 rods made from hollow carbon pipes or solid wood, steel, or aluminum. The rods varied in length, diameter, and material (see the Appendix). To prevent rod identification from these characteristics, identical $11.5-\mathrm{cm}$ plastic handles were attached to each rod. We were primarily interested in the mechanical variables $M$ and $I_{1}$. For novice perceivers, $M$ has been found to underlie length judgments during horizontal holding, and both $I_{1}$ and $M$ were implied during horizontal wielding, at least when the mechanical variables are computed with respect to the end of the rod (Kingma et al., 2004; van de Langenberg et al., 2006). ${ }^{2}$ The

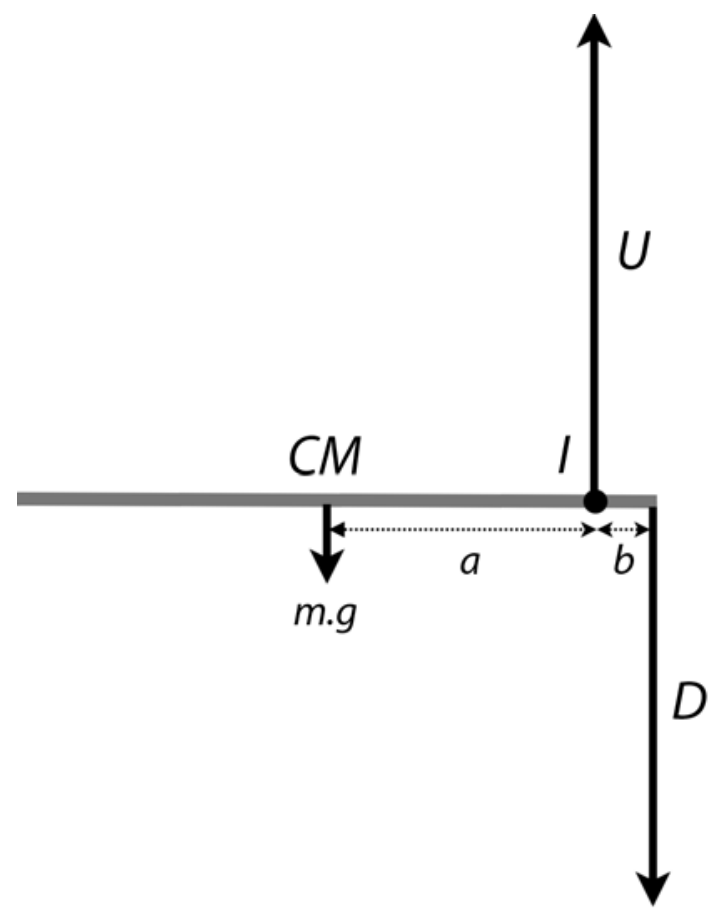

Figure 1. Depiction of the force gradient when a rod is held horizontally. The upward force $U$ at $I$ (the index finger) and the downward force $D$ (at the heel of the hand) are applied to one side of the center of mass $(C M)$. To maintain static equilibrium, it is required that $\sum F=0$ and $\sum M=0$. Calculating the equations relative to point $I$ gives $m g+D=U$ and $a . m g=b . D$, respectively. 
rods were chosen so that for each set actual length correlated weakly with the nonspecifying variables $I_{1}$ and $M$ (see Table 1). The reason for this was twofold. First, in both the test phases and the feedback phases, we tried to determine whether participants relied on a specifying or a nonspecifying mechanical variable. This is easier to do if these variables are disentangled - that is, if they correlate weakly with each other. Second, in the feedback phases, we intended to induce the perceptual learning process - that is, the convergence on specifying information. Earlier studies have revealed that this process is more likely to occur if the variable that participants initially detect correlates weakly with the to-be-perceived property (Jacobs et al., 2001; Michaels et al., 2008; Withagen \& Michaels, 2005). After all, reliance on such a variable yields substantial errors in the judgments, informing the participants in the feedback phase that a change in what information is exploited is required.

Design and Procedure. The experiment consisted of a pretest, four feedback blocks, and a posttest. The experiment was conducted over the course of 2 consecutive days. The first day consisted of a pretest and two feedback blocks, the second day of two feedback blocks followed by a posttest. In each block, the participants were to estimate the length of the handheld rod. As in earlier studies of length perception by dynamic touch (e.g., Kingma et al., 2004; Solomon \& Turvey, 1988; Withagen \& Michaels, 2004, 2005), the participant was seated on a stool, with the right forearm on an armrest. An opaque curtain between the stool and armrest concealed the handheld rod. On a table in front of the participant was a small planar surface that the participant could move along a rail by rotating a wheel with the left hand. The participant was to position the surface so that it coincided with the perceived distal end of the rod. The distance between the surface and the participant's hand was defined as perceived length.

Each block consisted of 26 trials. Each rod was offered twice in a random order. To hand the rods to the participants, we adopted the bimanual technique of van de Langenberg et al. (2006). The experimenter placed two fingers underneath either end of the rod. The rod handle was then placed into the waiting participant's hand, after which the weight of the rod was slowly shifted from the experimenter's fingers to the participant's hand. Great care was taken to avoid sudden shifts in rod position. In the test phases, the participants were instructed to hold the rod motionless at all times; obviously, however, small movements and tremor could not be completely avoided. In the literature on dynamic touch, it has been suggested that a small tremor might be sufficient to detect the rod's moments of inertia (Carello, Santana, \& Burton, 1996; but see Kingma et al., 2002). How much movement is needed to detect the moments of inertia is, of course, an empirical question. However, we consider it unlikely that they are accessible in our holding condition. We closely modeled both this condition and the technique of handing the rods on van de Langenberg et al.'s study. And although moments of inertia are always implied in length judgments while the rod is wielded, they were not while the rod was held horizontally. Hence, we believe that our holding context differs from our wielding context in that the moments of inertia are not available in the former but are in the latter.

Table 1

Correlations Between Logarithms of Candidate Variables and Actual Length

\begin{tabular}{|c|c|c|c|}
\hline & Length & $I_{1}$ & $M$ \\
\hline \multicolumn{4}{|c|}{ Test Rod Set } \\
\hline Length & - & .185 & -.138 \\
\hline$I_{1}$ & - & - & .948 \\
\hline$M$ & - & - & - \\
\hline \multicolumn{4}{|c|}{ Feedback Rod Set } \\
\hline Length & - & .117 & -.212 \\
\hline$I_{1}$ & - & - & .946 \\
\hline$M$ & - & - & - \\
\hline
\end{tabular}

In the feedback phases, the participants were to wield the rods. Because changes in information usage are likely to be accompanied by changes in the wielding behavior (cf. Riley, Wagman, Santana, Carello, \& Turvey, 2002), the participants were allowed to hold the rod loosely and wield it freely, with the exception that touching the curtain or the floor was prohibited. After the participant made the length judgment, we provided feedback on the actual length of the rod; we repositioned the planar surface so that it coincided with the actual distal end of the handheld rod (see Withagen \& Michaels, 2004, 2007). In both the test and feedback phases, the sliding surface had to be positioned at the proximal end of the scale after each trial. There was a short pause between the blocks. In the control group, the feedback phase was identical to that of the experimental group, except that no feedback was provided after the judgment of length.

\section{Results and Discussion}

We first tested whether the participants generally improved in their length judgments during the feedback phases (i.e., in the wielding context). We computed the Pearson product-moment correlations between perceived length and actual length for each participant in the first and the last feedback block. In the experimental group, the correlations averaged ${ }^{3}$ over participants changed from .387 in the first feedback phase to .708 in the last feedback phase. In the control group, the average correlations were .068 in the first feedback block and .166 in the last feedback block. ${ }^{4}$ We conducted a repeated measures ANOVA with the within-subjects factor of test (2 levels: Feedback Block 1 and Feedback Block 4) and the betweensubjects factor of group (2 levels: experimental, control). The ANOVA revealed a significant main effect of test $[F(1,10)=16.32, p<.01]$, indicating that participants generally improved in their judgments of length in the wielding context. The groups differed significantly from each other $[F(1,10)=21.61, p<.001]$. The significant interaction effect of group $\times$ test $[F(1,10)=6.92, p<$ $.05]$ indicates that the improvement in the judgments was significantly different between the two groups. An additional paired $t$ test demonstrated that, in the experimental group, a significant improvement in the length judgments occurred in the feedback phase $[t(7)=4.99, p<.01]$. This is in line with earlier studies that showed that perceptual learning occurs after feedback in the wielding condition (Michaels et al., 2008; Withagen \& Michaels, 2005).

To test whether the learning effect in the wielding context transferred to the holding context, we compared the correlations of perceived length and actual length on the pretest with the correlations of perceived length and actual length on the posttest. In the experimental group, the correlations averaged over participants were .190 in the pretest and .286 in the posttest. In the control group, the average correlations were .108 and .086 , respectively. A repeated measures ANOVA with the within-subjects factor of test ( 2 levels: pretest and posttest) and the between-subjects factor of group (2 levels: experimental, control) revealed no significant effects $(p s>.05)$. A separate analysis of the experimental group also showed no significant improvement in the length judgments $[t(7)=1.00, p>.05]$. Hence, perceived length was not more closely tied to actual length in the posttest than in the pretest; that is, no learning occurred. 
This absence of significant effects, however, might have been the result of large individual differences in the learning trajectories. Indeed, an earlier study revealed substantial individual differences in learning to perceive length by dynamic touch (Withagen \& van Wermeskerken, 2009; see also Jacobs et al., 2000; Jacobs et al., 2001; Michaels \& de Vries, 1998; Runeson \& Andersson, 2007; Runeson et al., 2000, for earlier portrayals of individual differences in other paradigms). A recent evolutionary analysis of perception and the use of information (Withagen \& Chemero, 2009) suggested that the search for such individual differences should be more central in the study of perception. ${ }^{5}$ Indeed, contemporary evolutionary theory does not warrant the assumption of a universal human perceptual apparatus. In fact, between-subjects variation in the use of information and in learning to perceive is to be expected. Hence, it might be more informative to investigate individual performances than to average over participants in the data analyses. To determine whether individual participants changed in what information they exploited, we used the method that was developed by Jacobs et al. (2000; see also Withagen \& Michaels, 2005; Withagen \& van Wermeskerken, 2009). For each individual and phase, we computed the correlations of perceived length with actual length and the candidate nonspecifying variables $I_{1}$ and $M$. The correlation between perceived length and actual length is equivalent to the correlation between perceived length and the specifying variables (e.g., Hoisington's [1920] force gradient, $\left.I_{1} / M, I_{1} / m, M / m\right)$. After all, specifying variables, by definition, relate one-to-one to the to-be-perceived property. However, because there are multiple specifying variables that could be exploited and we did not want to suggest that one of them is used, we decided to compute and report the correlation of perceived length with actual length.

In computing the correlations of perceived length with actual length and the variables $I_{1}$ and $M$, we used the logarithms of each of these variables because the relationships between perceived length and the nonspecifying variables $I_{1}$ and $M$ are expected to be nonlinear. To determine whether a participant relied on a specifying or a nonspecifying variable, we compared for each phase the correlation of perceived length and actual length with the correlation of perceived length and the most highly correlated nonspecifying variable $\left(I_{1}\right.$ or $\left.M\right)$. We performed a $t$ test for dependent correlations (Bruning \& Kintz, 1987, p. 228) to find out whether the difference between these two correlations was significant. A significant difference means that one informational variable explains the variance in perceived length significantly better than the other variable does. We concluded that the participant relied on a specifying or a nonspecifying variable (depending on which of the correlations was higher) only in the case of a significant difference between the two correlations.

The individual results of the experimental group are presented in Figure 2, which depicts the correlation of perceived length with actual length (i.e., a specifying variable) and the nonspecifying variables $I_{1}$ and $M$. More precisely, it portrays for each individual the extent to which these different variables can explain the variance in perceived length in the different blocks of the experiment. All but 1 participant (Participant 2) relied on a nonspecifying variable in the pretest - that is, in the holding condition. This is in line with earlier studies that demonstrated that length perception during forward holding is constrained by the nonspecifying variable $M$ (Kingma et al., 2004; van de Langenberg et al., 2006). In the feedback phases of the experiment, all participants changed in information usage. Participants 5 and 7, however, did not learn to detect specifying information. Participants 2, $3,4,6$, and 8 , on the other hand, did succeed in exploiting such information; in at least one feedback phase, the correlation between perceived length and actual length was significantly higher than was the correlation of perceived length with the most highly correlated nonspecifying variable. Participant 1 showed a nonsignificant trend toward relying on specifying information. As previously mentioned, convergence on specifying information in the wielding context had been demonstrated earlier (Withagen \& Michaels, 2005; Withagen \& van Wermeskerken, 2009). Furthermore, and also in line with the earlier studies, there were substantial individual differences in the learning trajectories. The participants varied in how quickly they learned to detect specifying information and how firmly they maintained reliance on such information. As mentioned earlier, inspired by an evolutionary analysis of perception (Withagen \& Chemero, 2009), Withagen and van Wermeskerken took these differences as evidence for variation in the perceptual apparatuses of members of the human species.

Individual differences were also apparent when the participants returned to the holding context in the posttest. Participants 4, 5, and 7 returned to the use of nonspecifying information in the posttest. Hence, although feedback had led to changes in information usage in the wielding condition (especially for Participant 4), these changes disappeared in the holding condition - in both the pretest and the posttest, these participants detected a nonspecifying variable. This is in line with van de Langenberg et al.'s (2006) salience hypothesis that states that in the horizontal holding condition, $M$ is salient and will therefore be used. The other participants, however, did not return to the use of a nonspecifying variable, so for them, the feedback in the wielding condition changed the information usage in the holding condition. It is interesting to note, however, that none of the learners reliably detected specifying information in the posttest. Participants $1,3,6$, and 8 showed only a nonsignificant use of a specifying mechanical variable. However, the correlation of perceived length with this variable is substantially lower in the posttest than during the feedback phases. The fact that participants did not succeed in reliably detecting specifying information in the posttest might not be remarkable. After all, the participants might have learned to rely on the specifying variable $I_{1} / M$ in the wielding condition, and moments of inertia are not available in the holding condition that we contrived (see Kingma et al., 2004; van de Langenberg et al., 2006). What is remarkable, though, is that 5 of the 8 participants did not exploit the nonspecifying variable they started with. Hence, al- 

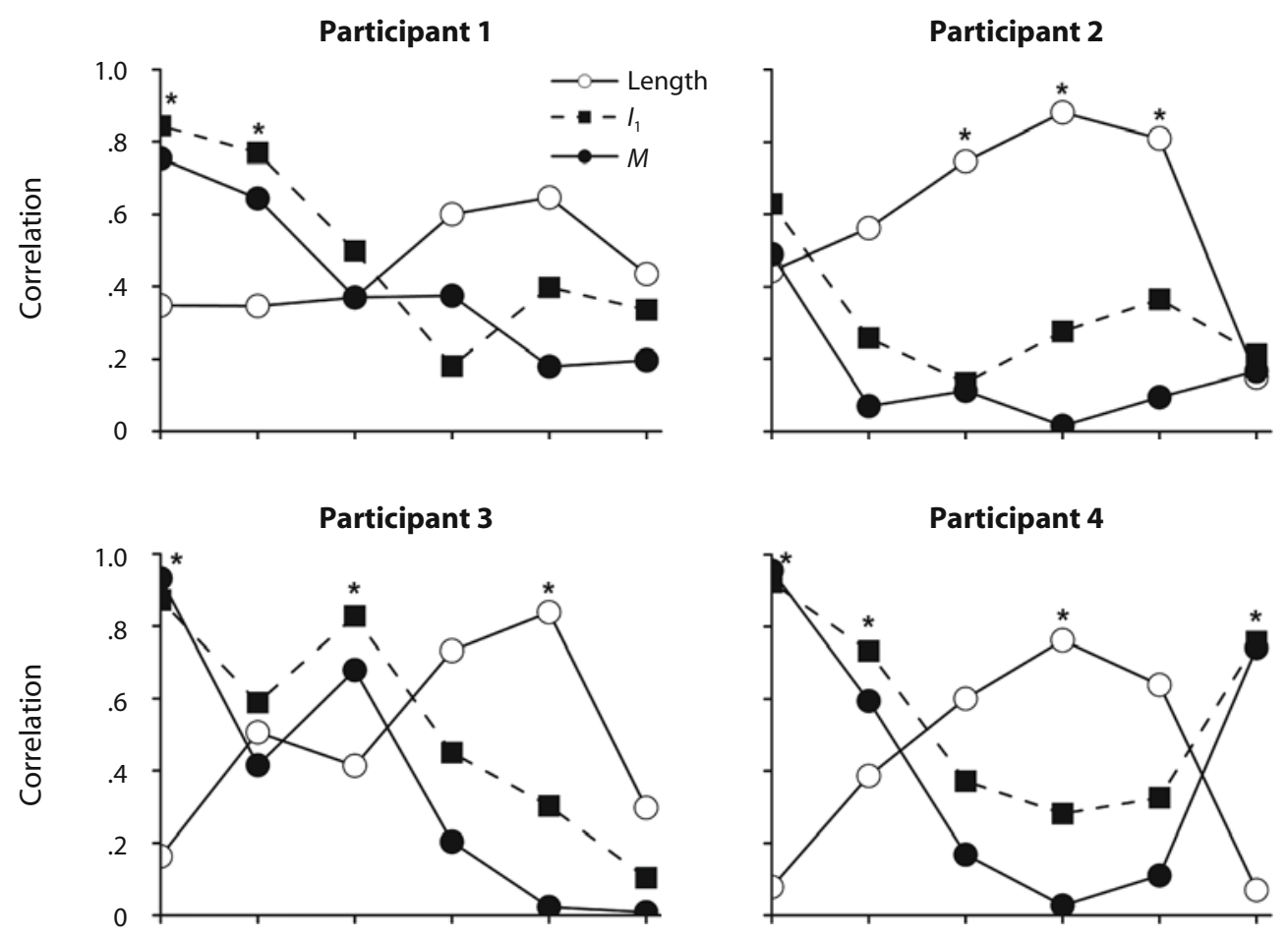

Participant 4

Participant 5
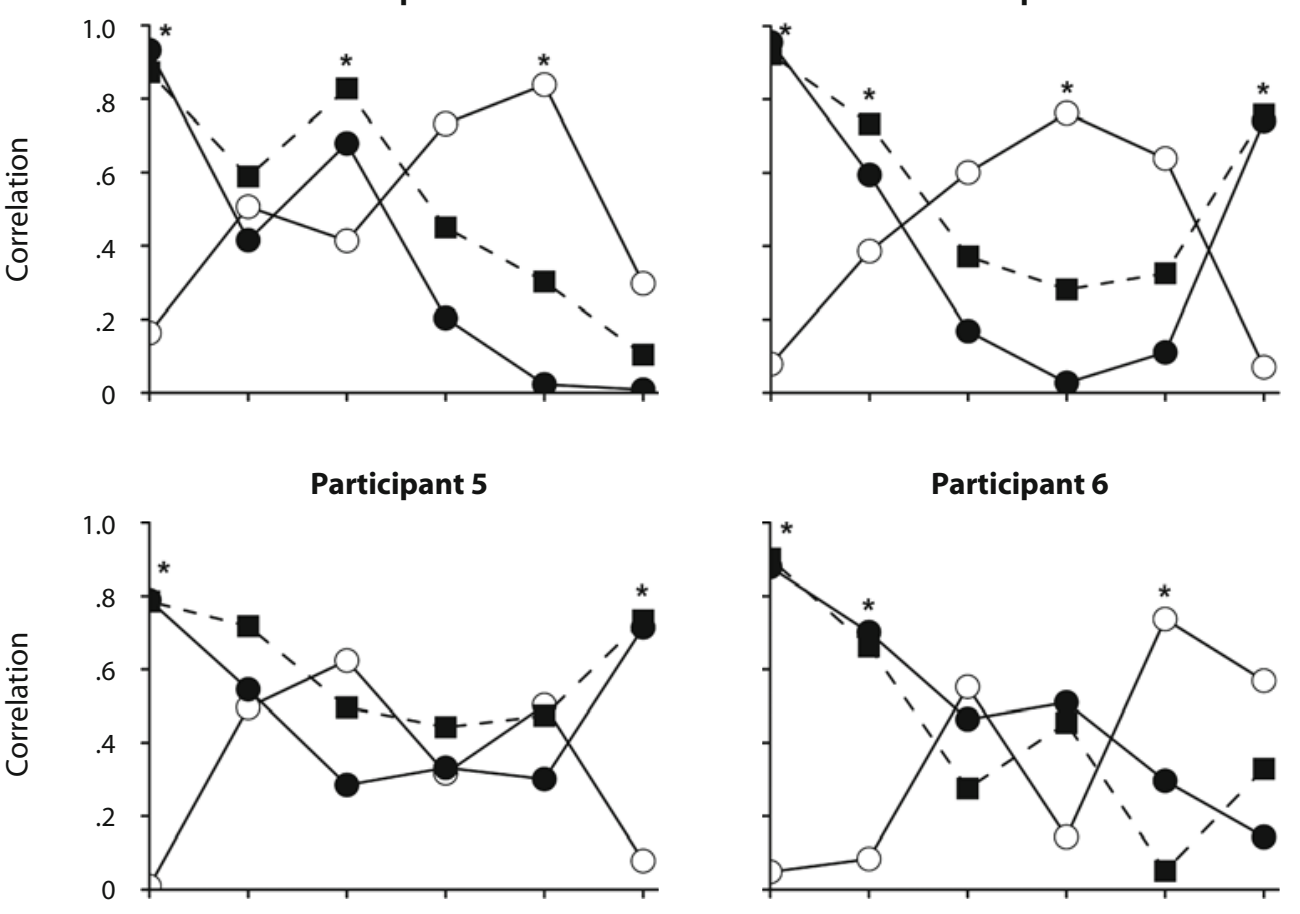

Participant 6

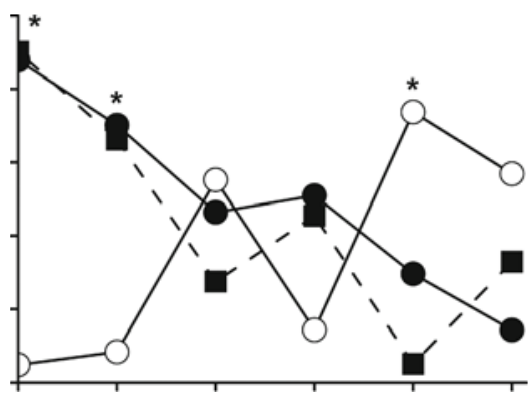

Participant 7
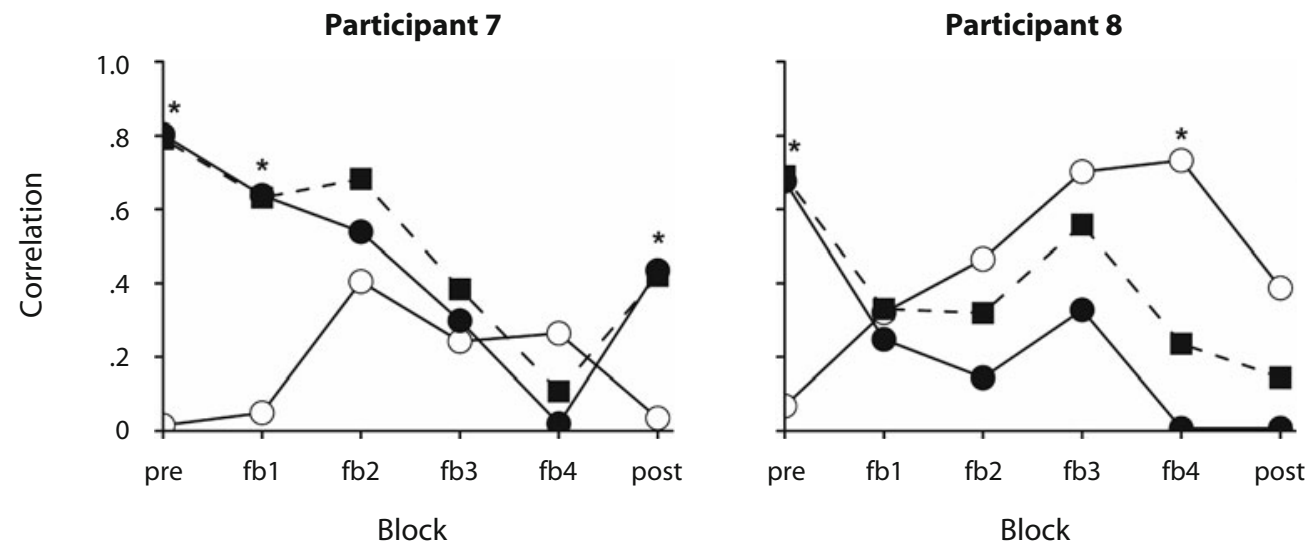

Figure 2. Correlations between the length judgments and $I_{1}, M$, and actual length for the experimental group in Experiment 1. In the blocks marked with an asterisk, there is a significant difference $(p<.05$, two-tailed) between the correlation of perceived length with actual length and the correlation of perceived length with the most highly correlated nonspecifying variable. pre, pretest; fb, feedback phase; post, posttest. 
though these participants have not succeeded in reliably detecting the specifying variable in the holding context, they have unlearned to exploit the nonspecifying variable they initially used. Apparently, perceivers do not simply rely on the mechanical variable that is most salient in the stimulus flow. For several perceivers, what information is detected in the holding context also seems to depend on their learning history, even if feedback was provided in the wielding condition.

The individual results of the control group are shown in Figure 3. All participants relied on nonspecifying variables during the whole experiment. Hence, changing mechanical context and experience with the task does not lead to a convergence on specifying information. This indicates that the changes in information usage observed in the experimental group were the result of feedback.

\section{EXPERIMENT 2}

Experiment 2 had two goals. The first goal was to test whether perceivers can learn to exploit specifying information in the horizontal holding condition. As mentioned in the introduction, this had not been examined before. However, perceptual learning is possible, because in this condition there are also mechanical variables available that specify the length of homogeneous rods. The second goal was to test whether the possible learning effects in the holding condition transfer to the wielding condition. Because the specifying variables in the holding context are available while the rod is wielded, we expected possible learning effects to transfer across mechanical contexts. The experiment had the same design as Experiment 1, except that participants were to wield the rods in the test phases and to hold the rods in the feedback phases. As in Experiment 1, there was an experimental group and a control group. The former received visual information about actual rod length in the feedback phases; the latter received no feedback at all.

\section{Method}

Participants and Materials. Twelve new participants (10 men and 2 women) volunteered to participate; each gave their informed consent. Their ages ranged from 19 to 22 years. One participant was left-handed; 11 were right-handed. As in Experiment 1, 8 participants were assigned to the experimental group, 4 to the control group. The experimental setup and the rod sets were the same as in Experiment 1.

Design and Procedure. We used the same experimental design as in Experiment 1, except that participants were to wield the rods in the test phases and to hold them in the feedback phases. In the feedback trials, the participants were to hold the rod motionless at all times. After a participant estimated the length, we repositioned the planar surface so that it was aligned with the actual distal end of the rod.

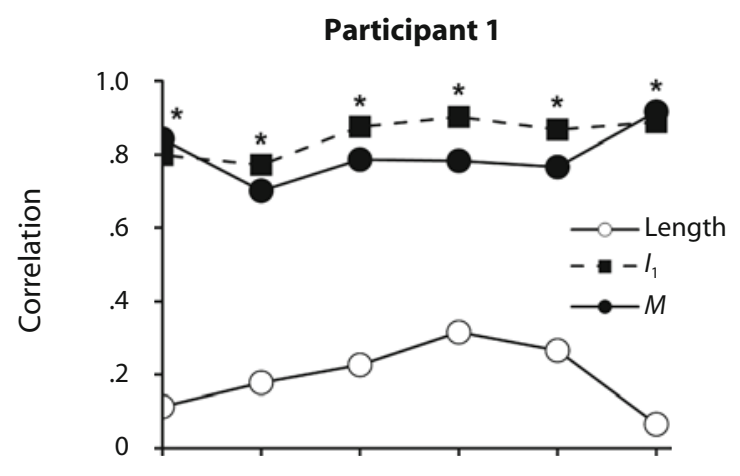

Participant 3

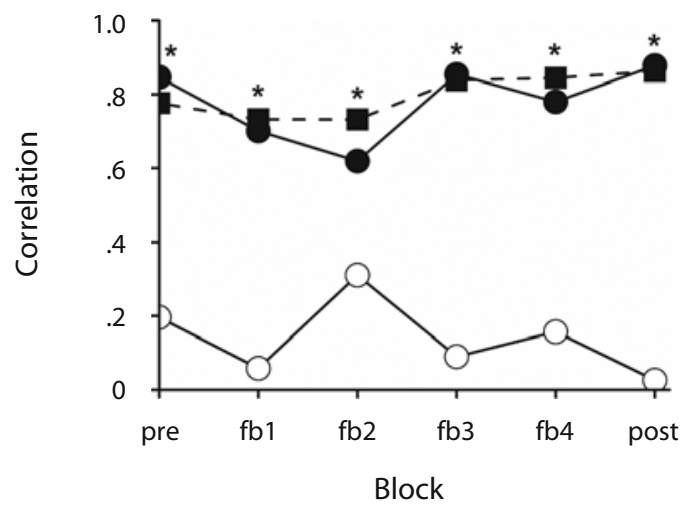

Participant 2

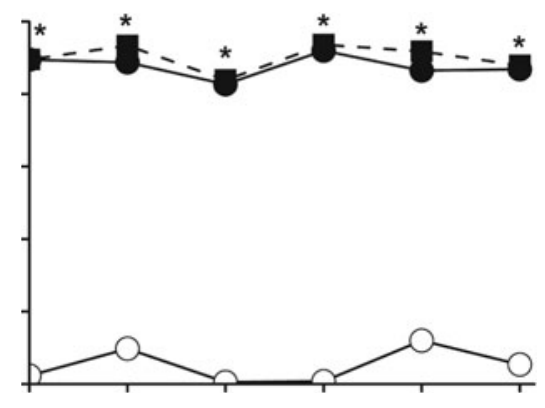

Participant 4

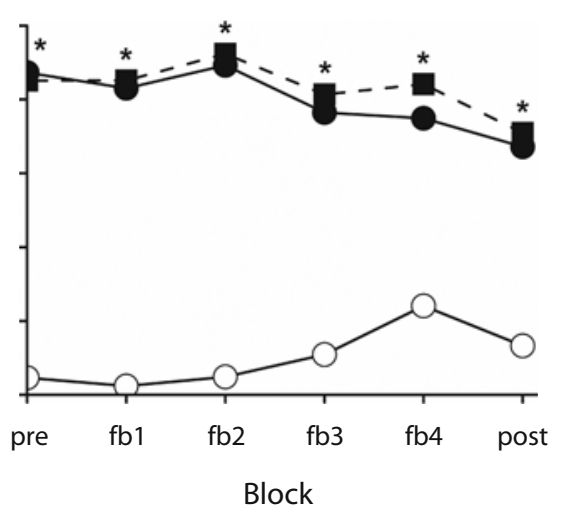

Figure 3. Correlations between the length judgments and $I_{1}, M$, and actual length for the control group in Experiment 1. In the blocks marked with an asterisk, there is a significant difference $(p<.05$, two-tailed) between the correlation of perceived length with actual length and the correlation of perceived length with the most highly correlated nonspecifying variable. pre, pretest; fb, feedback phase; post, posttest. 


\section{Results and Discussion}

We were first interested in whether perceptual learning occurred in the feedback phases. As mentioned earlier, perceptual learning in the horizontal holding context had not been examined before. As in Experiment 1, we computed the correlations of perceived length and actual length for each participant in both the first and the last feedback block. In the experimental group, the correlations averaged over participants were .153 in the first feedback phase and .348 in the last feedback phase. For the control group, the average correlations were .178 and .148 , respectively. A repeated measures ANOVA with the within-subjects factor of test (2 levels: Feedback Block 1, Feedback Block 4) and the between-subjects factor of group (2 levels: experimental, control) revealed only a significant interaction effect of test $\times$ group $[F(1,10)=$ $6.54, p<.05]$. This suggests that the changes in the length estimates differed between the two groups. A separate analysis revealed that for the experimental group, the length judgments were more closely tied to actual length in the last feedback phase than in the first feedback phase $[t(7)=3.33, p<.05]$. Apparently, learning did occur in the holding condition.

Also, when we compared posttest with pretest judgments, a significant learning effect showed up. Again, we computed the correlations between the length judgments and actual length for each participant and each test phase. For the experimental group, the correlations averaged over participants changed from .247 in the pretest to .555 in the posttest. In the control group, the average correlations were .238 in the pretest and .233 in the posttest. A repeated measures ANOVA with the within-subjects factor of test (2 levels: pretest, posttest) and the between-subjects factor of group (2 levels: experimental, control) revealed a significant effect of test $[F(1,10)=5.93, p<.05]$; the perceptual judgments were more closely tied to actual length in the posttest than in the pretest. The groups did not differ from each other $[F(1,10)=4.31, p>.05]$. The significant interaction effect of group $\times$ test $[F(1,10)=6.33, p<$ $.05]$ indicates that the change in the judgments is the result of feedback. An additional analysis of the experimental group showed that the correlation with actual length in the posttest was significantly higher than in the pretest $[t(7)=$ $4.23, p<.01]$. Apparently, and in line with our hypothesis, the changes in information usage in the holding context also manifest themselves in the wielding condition.

As in Experiment 1, we investigated the changes in information usage for each individual. As mentioned earlier, individual performances provide an insightful picture of the learning process (Jacobs et al., 2000; Jacobs et al., 2001; Michaels \& de Vries, 1998; Runeson \& Andersson, 2007; Runeson et al., 2000; Withagen \& Michaels, 2005; Withagen \& van Wermeskerken, 2009). We calculated the correlations between the logarithms of perceived length and actual length, $I_{1}$, and $M$ for each participant and each phase. Figure 4 presents the results of the experimental group. All but 2 participants (Participants 3 and 5) detected nonspecifying information in the pretest. This is in keeping with earlier studies that demonstrated that in the wielding condition, novice perceivers tend to rely on the nonspecifying mechanical invariants $I_{1}$ and/or $M$ (Kingma et al., 2004; van de Langenberg et al., 2006; Withagen \& Michaels, 2005; Withagen \& van Wermeskerken, 2009). In the feedback phases, all participants changed in information usage, but also in this experiment there were substantial individual differences. Participant 5 learned to detect specifying information - in the second feedback block, the correlation of perceived length with actual length is significantly higher than is the correlation of perceived length with the most highly correlated nonspecifying variable. Although this learning effect diminished in later feedback blocks, it was of interest, because learning to exploit specifying information in the horizontal holding context had not been demonstrated before. Participants 3 and 7 demonstrated nonsignificant trends to rely on specifying information. The other participants did not learn to detect the mechanical invariant that specifies length. However, they did not stick to the use of the nonspecifying variable they started with.

The fact that feedback in the holding condition led to changes in the participants' perceptual apparatuses is also apparent when we look at the individual performances in the posttest - that is, in the wielding condition. None of the participants returned to the use of the nonspecifying variable they started with. In fact, for each and every participant the correlation of perceived length with actual length is higher than is the correlation of perceived length with the nonspecifying variables, although for none of the individual participants is this difference significant. However, when we averaged over participants in the experimental group, the correlation with actual length (.544; i.e., a specifying variable) is significantly higher than the correlation with $I_{1}(.245)$, the most highly correlated nonspecifying variable $[t(7)=4.67, p<.01]$. Furthermore, it is interesting to note that for each and every participant the correlation of perceived length and actual length increased when they moved from the holding condition to the wielding context. This might suggest that participants learned to exploit a mechanical variable in the holding context that is easier to detect in the wielding condition.

The individual results of the control group are depicted in Figure 5. As in Experiment 1, the participants generally relied on nonspecifying information during the whole study. Hence, neither changing the mechanical context, nor having experience with judging length, induced the learning process. Again, this suggests that the observed changes in information usage in the experimental group are the result of feedback.

\section{GENERAL DISCUSSION}

In the experiments reported here, we tested how feedback and mechanical context jointly determine the use of mechanical variables in perceiving length by dynamic touch. Earlier studies have revealed that both feedback and mechanical context constrain what mechanical invariant is exploited (Kingma et al., 2004; Michaels et al., 2008; van de Langenberg et al., 2006; Wagman et al., 2001; Withagen \& Michaels, 2005; Withagen \& van Wermeskerken, 2009). However, these studies have focused on 

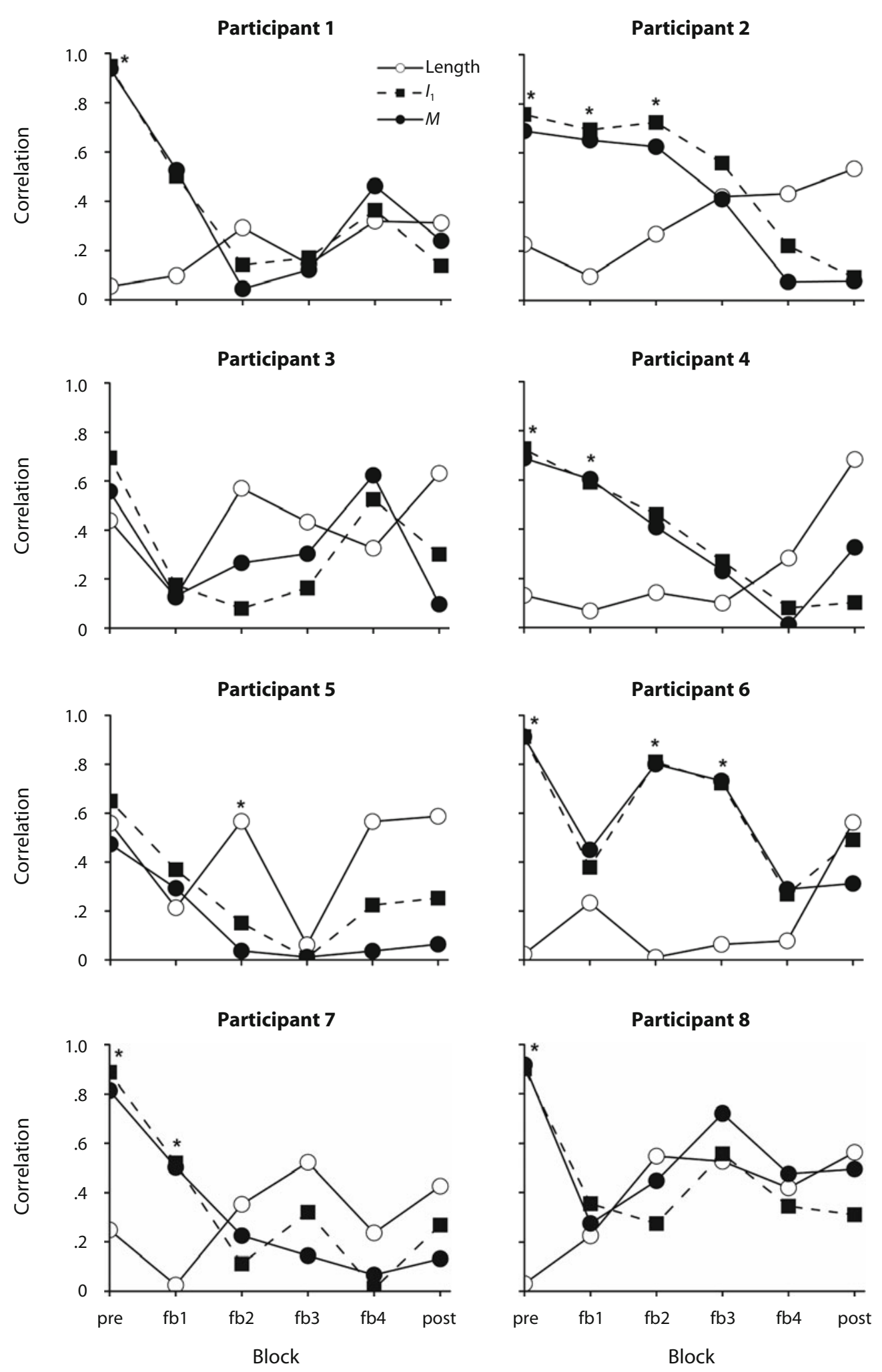

Figure 4. Correlations between the length judgments and $I_{1}, M$, and actual length for the experimental group in Experiment 2. In the blocks marked with an asterisk, there is a significant difference $(p<.05$, two-tailed) between the correlation of perceived length with actual length and the correlation of perceived length with the most highly correlated nonspecifying variable. pre, pretest; fb, feedback phase; post, posttest. 


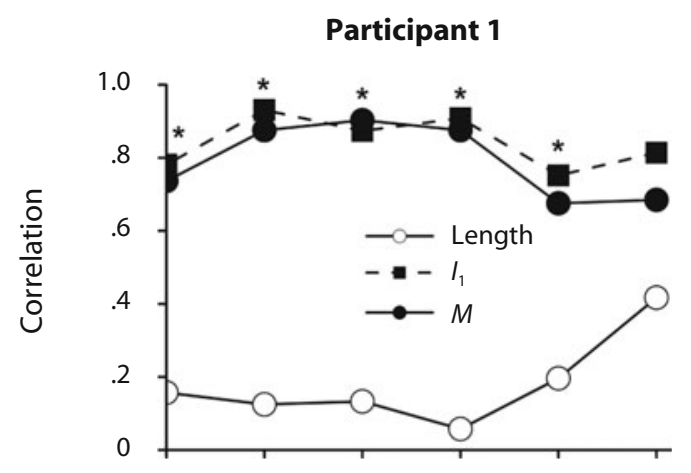

Participant 3

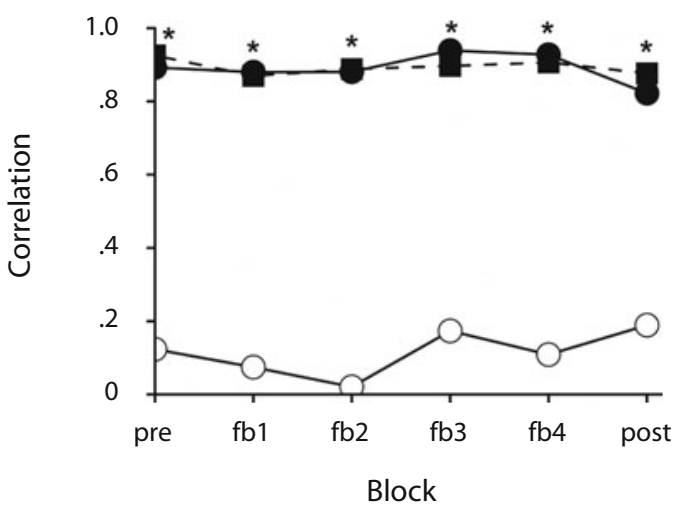

Participant 2

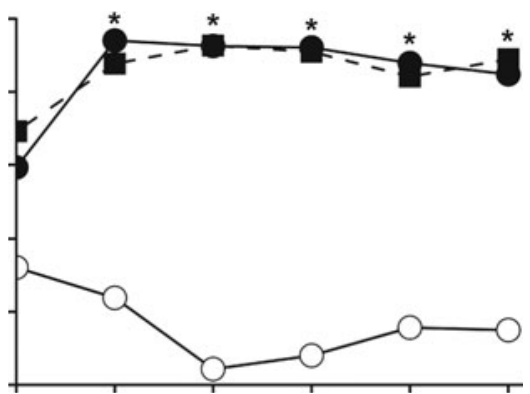

Participant 4

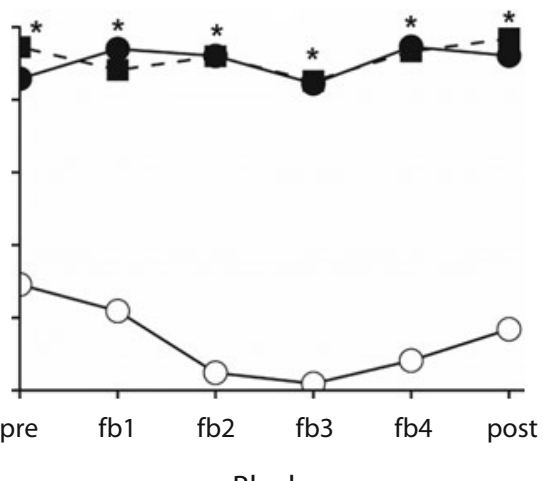

Block

Figure 5. Correlations between the length judgments and $I_{1}, M$, and actual length for the control group in Experiment 2. In the blocks marked with an asterisk, there is a significant difference $(p<.05$, two-tailed) between the correlation of perceived length with actual length and the correlation of perceived length with the most highly correlated nonspecifying variable. pre, pretest; fb, feedback phase; post, posttest.

the effects of a single factor on information usage. The present study is the first to examine how feedback and mechanical context jointly influence what informational variable is exploited in perceiving length by dynamic touch. We conducted two experiments, each with a standard pretest-feedback-posttest design. In Experiment 1, participants were to hold the rods horizontally in the test phases and wield the rods in the feedback phases. Participants generally relied on nonspecifying information in the pretest. As did the authors of an earlier study (Withagen \& Michaels, 2005), we found that after feedback most participants learned to detect specifying information in the wielding condition. However, 3 of the 8 participants returned to the use of nonspecifying information in the posttest - that is, in the holding condition. The information usage of the other 5 participants changed, but none of them reliably detected specifying information while holding the rod stationary. In Experiment 2, we reversed the mechanical contexts: Participants were to wield the rods in the test phases and to hold the rods horizontally in the feedback phases. Participants generally detected nonspecifying information while wielding the rod in the pretest. After being provided with feedback in the holding condition, all participants changed in variable usage, but only 1 participant succeeded in detecting the specifying information. The changes in information usage were also manifest in the wielding condition of the posttest. In general, the length judgments were more closely tied to actual length in the posttest than in the pretest. In fact, none of the participants returned to the exploitation of nonspecifying information they had started with.

The remainder of the article consists of two sections. In the first, we explore the implications of our findings for discussions on perceptual learning in dynamic touch. In the second, we examine the consequences for the salience hypothesis of van de Langenberg et al. (2006).

\section{Perceptual Learning in Dynamic Touch}

As previously mentioned, earlier studies have already examined perceptual learning in dynamic touch (Michaels et al., 2008; Wagman et al., 2001; Withagen \& Michaels, 2005; Withagen \& van Wermeskerken, 2009). These studies have revealed that after feedback perceivers can change the mechanical variables they exploit to perceive length. Participants generally rely on a nonspecifying mechanical invariant in the pretest but can learn to detect the more useful information after feedback. However, a recent study demonstrated substantial individual differences in learning to perceive length by dynamic touch (Withagen \& van Wermeskerken, 2009). Perceivers were found to vary in whether, when, and how they responded to feedback. What does the present study add to this earlier work? 
Up to this point, studies of learning to perceive length by dynamic touch all studied this process in the horizontal wielding condition. In the present study, we also examined whether learning can occur while holding the rod horizontally. As mentioned in the introduction, in this condition there are mechanical variables available that specify the length of homogeneous rods. Indeed, as already suggested by Hoisington (1920), the force gradient given by the ratio of the downward and upward forces on the hand relates one-to-one to length. Also, the ratio of $M$ and $m$ is specific to length. We found that, for the experimental group, the judgments in the last feedback phase were more closely tied to actual length than were the judgments in the first feedback phase. However, as in the wielding condition, there were substantial individual differences in learning to perceive length in this context. One participant learned to detect the specifying information in the feedback blocks; 2 other participants showed nonsignificant trends to rely on a specifying variable. Hence, learning to exploit specifying information while holding a rod horizontally is possible. However, one might wonder why only 1 of the 8 participants succeeded in learning to exploit the specifying information in this condition.

As previously mentioned, inspired by an evolutionary analysis of perception (Withagen \& Chemero, 2009), Withagen and van Wermeskerken (2009) recently explained individual differences in learning performances in terms of variation in perceptual learning capacities, and suggested a possible mechanism. As argued by Runeson and colleagues (Runeson \& Andersson, 2007; Runeson et al., 2000), in order to learn, a perceiver must be capable of separating the noise in the perceptual system (Thurstonian error) from the error that is the result of the detection of nonspecifying information (Brunswikian error). Withagen and van Wermeskerken surmised that perceivers might vary in the capacity to distinguish these two types of error, meaning that perceivers vary in how well and how quickly they can learn perceptual tasks. It is not unlikely that variation in this capacity also accounts for some of the observed individual differences in learning in both the wielding and the holding context. However, another explanation might be that perceivers vary in their capacity to detect mechanical variables. ${ }^{6}$ From an evolutionary perspective, it is to be expected that perceivers not only vary in their capacity to learn, but also in their capacity to detect certain informational variables. In addition, it has been argued that one cannot assume that all perceptual variables are detectable by the perceptual system (Withagen, 2004; Withagen \& Chemero, 2009). Because of the many constraints on the evolutionary and developmental processes, it might be that the anatomical structures required for the (proper) detection of certain information cannot evolve. Hence, it might be that the aforementioned force gradient or $M / m$ is not (easily) detectable, and/or that perceivers might vary in their capacity to do so. This suggested variation in the capacity to detect mechanical variables might explain that, whereas all participants changed in what information they detected after feedback in the holding context, only 1 succeeded in discovering the specifying mechanical variable. Furthermore, the idea that the force gradient and/or $M / m$ are not easily detectable also explains the relatively low correlations of the perceptual judgments with actual length in the feedback blocks of Experiment 2 and the posttest of Experiment 1. Given the omnipresent variation in the human species, it is quite likely that variation in both the capacity to learn and the capacity to detect certain mechanical variables contributes to the observed individual differences in learning to perceive length by dynamic touch. However, further research is needed to settle this issue.

A second contribution of the present study to the literature on learning in dynamic touch lies in the examination of information usage in another mechanical context. Indeed, both experiments were designed to address the question of whether learning effects in one mechanical condition transfer to another mechanical context. We found that in general changes in information usage induced by the feedback were not confined to the mechanical context in which the feedback was provided. However, primarily in Experiment 1, there were also individual differences in transfer of learning effects. In this experiment, participants returned to the holding context after being provided with feedback while wielding the rod. Three of the 8 participants returned to the detection of nonspecifying information in the posttest. For the other 5 participants, the feedback in the wielding condition led to changes in information usage in the holding condition. At present, it is unclear how to account for these differences in transfer. Apparently, they cannot be explained in terms of the aforementioned variation in learning capacity or in the ability to detect mechanical variables. Our best bet is that the individual differences in transfer have something to do with the variable(s) the participants had learned to detect in the holding condition. Recall that there is an asymmetry in the information available in the wielding context and the holding context. Moments of inertia are detectable while wielding a rod but not while holding it stationary - at least in the holding condition that we contrived (Kingma et al., 2002; van de Langenberg et al., 2006). Hence, not all the mechanical variables available in the wielding condition are present in the holding condition, but the reverse is true: all the variables present while a rod is being held stationary are available while it is wielded. It might be that participants who learned to rely on a variable in the wielding condition not available in the holding context returned in the posttest to the nonspecifying variable they had started with. On the other hand, when participants learned to exploit a variable in the wielding context detectable in the holding condition (even if this is not easy), they might stick to this variable in the posttest and not return to the variable they initially detected. Of course, further research is needed to test this rather speculative hypothesis.

However, the fact that there were no individual differences in transfer in Experiment 2 is consonant with this idea. In this experiment, participants were to wield the rods after being provided with feedback in the holding context. In the posttest, none of the participants demonstrated the return to the informational variable they relied on in the pretest. In fact, the correlation of perceived length with actual length (i.e., a specifying variable) was higher than 
was the correlation of perceived length with the nonspecifying variables, so the participants have probably learned to rely on a variable in the holding context available in the wielding condition also. In fact, the universal increase in correlation with actual length when moving from the holding condition to the wielding condition suggests that this variable is easier to detect while the rod is wielded. In any case, the present study showed that feedback in one mechanical context brings about changes in the perceptual apparatus that often manifest themselves in another mechanical context. This brings us to the implications of our findings for the salience hypothesis introduced by van de Langenberg et al. (2006).

\section{Salience Hypothesis}

As mentioned in the introduction, van de Langenberg et al. (2006) studied length perception through dynamic touch in different mechanical contexts. Their idea was that mechanical invariants $\left(I_{1}, I_{3}, M, m\right)$ contributed to the stimulus flow as a function of the context in which the length had to be judged. Van de Langenberg et al. hypothesized that perceivers would rely on the mechanical invariants salient in that flow. For example, while a rod is held vertically, the only variable that determines the exerted force is $m$, and this variable is thus likely to be used to perceive length. When a rod is held horizontally, on the other hand, the torque needed at the endpoint is determined by $M$ alone. And when a rod is wielded horizontally, $I_{1}$ is an important factor in determining the stimulus flow, especially when the wielding speed is substantial. Van de Langenberg et al. found that perceivers indeed detected the mechanical invariants salient in the stimulus flow in a certain mechanical context.

In the discussion of their article, van de Langenberg et al. (2006) argued that their salience hypothesis was of interest for research on learning in dynamic touch. They suggested that because exploratory behavior (e.g., wielding speed and amplitude) determines the salience of informational variable in the stimulus flow, this behavior is likely to provide a window into the participant's use of mechanical variables and changes therein. It is indeed probable that in the wielding conditions of the present experiments, changes in information usage are reflected in the wielding behavior. Indeed, perceivers have been found to adjust their wielding behavior to the mechanical variable they intend to detect (Riley et al., 2002). However, the present study demonstrates that perceivers do not always rely on the salient variables in the array-changes in information usage are not always accompanied by changes in perceptual behavior. Indeed, in the holding conditions of both Experiment 1 and Experiment 2, most participants were found to change the informational basis of their length estimates - that is, when their exploratory behavior (i.e., holding a rod horizontally) remained the same. In fact, the only changes that might have occurred in this condition are small alterations to the tremor and the way the participants held the rod. However, such changes are not likely to change the (relative) salience of the different mechanical variables in the haptic array. Granted - and as mentioned above - it has been suggested in the literature that tremor might suffice to detect the rod's moments of inertia (e.g., Carello et al., 1996), and changes in tremor might thus reveal something about what information is detected. However, even if experimental studies reveal that tremor suffices to detect moments of inertia, these moments still have a relatively minor role in determining the stimulus array in the holding condition and are thus not salient. After all, compared with $M$, the contribution of $I_{1}$ to this array is rather small (Kingma et al., 2002). This means that the observed changes in information usage in the holding condition indicate that participants do not always detect the most salient mechanical variable in the stimulus flow. In fact, feedback seems to be capable of educating the perceiver's attention to less salient mechanical variables in the haptic array.

However, as we have seen, the effect of feedback on information usage is perceiver dependent; feedback has a different effect on different perceivers, indicating that there are also organismal factors involved in determining what information is exploited (Withagen \& van Wermeskerken, 2009). This proves once more that (changes in) information usage in dynamic touch cannot be explained in terms of a single factor (i.e., mechanical context or feedback). Indeed, the studies to date suggest that there are at least three components to the determination of what mechanical invariant is relied on: feedback, mechanical context, and perceiver characteristics. This brings us back to the main conjecture of this article: To develop a theory of perception, researchers should reveal how multiple environmental and organismal factors jointly determine what information is detected (Withagen \& Chemero, 2009).

\section{AUTHOR NOTE}

We are grateful to Raoul Bongers, Esther Smits, and Carlijn Vernooij for helpful comments on an earlier version of this article. At Hof and Suzanne Nooij are gratefully acknowledged for discussion on the mechanics. Correspondence concerning this article and reprint requests should be addressed to R. Withagen, Center for Human Movement Sciences, University of Groningen, P.O. Box 196, 9700 AD Groningen, The Netherlands (e-mail: r.g.withagen@rug.nl).

\section{REFERENCES}

Bruning, J. L., \& Kintz, B. L. (1987). Computational handbook of statistics (3rd ed.). Glenview, IL: Scott Foresman.

Burton, G., \& Turvey, M. T. (1990). Perceiving the lengths of rods that are held but not wielded. Ecological Psychology, 2, 295-324.

Carello, C., Fitzpatrick, P., Domaniewicz, I., Chan, T.-C., \& TurvEY, M. T. (1992). Effortful touch with minimal movement. Journal of Experimental Psychology: Human Perception \& Performance, 18, 290-302.

Carello, C., Santana, M.-V., \& Burton, G. (1996). Selective perception by dynamic touch. Perception \& Psychophysics, 58, 1177-1190.

Cutting, J. E. (1986). Perception with an eye for motion. Cambridge, MA: MIT Press

Cutting, J. E. (1991). Four ways to reject directed perception. Ecological Psychology, 3, 25-34.

Cutting, J. E., \& Millard, R. T. (1984). Three gradients and the perception of flat and curved surfaces. Journal of Experimental Psychology: General, 113, 198-216.

FAJEN, B. R. (2008). Perceptual learning and the visual control of braking. Perception \& Psychophysics, 70, 1117-1129.

Fajen, B. R., \& DeVAney, M. C. (2006). Learning to control collisions: The role of perceptual attunement and action boundaries. Journal of 
Experimental Psychology: Human Perception \& Performance, 32, 300-313.

Fitzpatrick, P., Carello, C., \& Turvey, M. T. (1994). Eigenvalues of the inertia tensor and exteroception by the "muscle sense." Neuroscience, 60, 551-568.

Gibson, E. J. (1991). Perceptual learning. In E. J. Gibson (Ed.), An odyssey in learning and perception. Cambridge, MA: MIT Press. (Reprinted from Annual Review of Psychology, 14, 29-56, 1963)

Gibson, J. J. (1966). The senses considered as perceptual systems. Boston: Houghton Mifflin.

Gibson, J. J., \& Gibson, E. J. (1955). Perceptual learning: Differentiation or enrichment? Psychological Review, 62, 32-41.

Hoisington, L. B. (1920). On the non-visual perception of the lengths of lifted rods. American Journal of Psychology, 31, 114-146.

INGOLD, T. (2000). Evolving skills. In H. Rose \& S. Rose (Eds.), Alas, poor Darwin: Arguments against evolutionary psychology (pp. 273298). London: Jonathan Cape.

Jacobs, D. M., Michaels, C. F., \& Runeson, S. (2000). Learning to perceive the relative mass of colliding balls: The effects of ratio scaling and feedback. Perception \& Psychophysics, 62, 1332-1340.

Jacobs, D. M., Runeson, S., \& Michaels, C. F. (2001). Learning to perceive the relative mass of colliding balls in globally and locally constrained task ecologies. Journal of Experimental Psychology: Human Perception \& Performance, 27, 1019-1038.

Kingma, I., BeeK, P. J., \& van DieËn, J. H. (2002). The inertia tensor versus static moment and mass in perceiving length and heaviness of hand-wielded rods. Journal of Experimental Psychology: Human Perception \& Performance, 28, 180-191.

Kingma, I., van de Langenberg, R., \& Beek, P. J. (2004). Which mechanical invariants are associated with the perception of length and heaviness of a nonvisible handheld rod? Testing the inertia tensor hypothesis. Journal of Experimental Psychology: Human Perception \& Performance, 30, 346-354.

Michaels, C. F., ArZAmarski, R., Isenhower, R. W., \& Jacobs, D. M. (2008). Direct learning in dynamic touch. Journal of Experimental Psychology: Human Perception \& Performance, 34, 944-957.

Michaels, C. F., \& DE VRies, M. M. (1998). Higher order and lower order variables in the visual perception of relative pulling force. Journal of Experimental Psychology: Human Perception \& Performance, 24, 526-546.

NISBETT, R. E. (2003). The geography of thought: How Asians and Westerners think differently . . and why. New York: Free Press.

Nisbetт, R. E., \& Miуamoto, Y. (2005). The influence of culture: Holistic versus analytic perception. Trends in Cognitive Sciences, 9, 467473.

Oyama, S. (1985). The ontogeny of information: Developmental systems and evolution. New York: Cambridge University Press.

Riley, M. A., Wagman, J. B., Santana, M., Carello, C., \& Turvey, M. T. (2002). Perceptual behavior: Recurrence analysis of a haptic exploratory procedure. Perception, 31, 481-510.

Runeson, S., \& Andersson, I. E. K. (2007). Achievement of specificational information usage with true and false feedback in learning a visual relative-mass discrimination task. Journal of Experimental Psychology: Human Perception \& Performance, 33, 163-182.

Runeson, S., Juslin, P., \& Olsson, H. (2000). Visual perception of dynamic properties: Cue heuristics versus direct-perceptual competence. Psychological Review, 107, 525-555.

Solomon, H. Y., \& Turvey, M. T. (1988). Haptically perceiving the distance reachable with hand-held objects. Journal of Experimental Psychology: Human Perception \& Performance, 14, 404-427.

Thelen, E., \& Smith, L. B. (1996). A dynamic systems approach to the development of cognition and action. Cambridge, MA: MIT Press.

Tresilian, J. R. (1999). Visually timed action: Time-out for "tau"? Trends in Cognitive Sciences, 3, 301-310.

Turvey, M. T. (1996). Dynamic touch. American Psychologist, 51, 1134-1152.

van de Langenberg, R., Kingma, I., \& Beek, P. J. (2006). Mechanical invariants are implicated in dynamic touch as a function of their salience in the stimulus flow. Journal of Experimental Psychology: Human Perception \& Performance, 32, 1093-1106.

VAN Hof, P., van DER Kamp, J., \& SAVElSbergh, G. J. P. (2006). Threeto eight-month-old infants' catching under monocular and binocular vision. Human Movement Science, 25, 18-36.

Wagman, J. B., Shockley, K., Riley, M. A., \& Turvey, M. T. (2001). Attunement, calibration, and exploration in fast haptic perceptual learning. Journal of Motor Behavior, 33, 323-327.

WithageN, R. (2004). The pickup of nonspecifying variables does not entail indirect perception. Ecological Psychology, 16, 237-253.

Withagen, R., \& Chemero, A. (2009). Naturalizing perception: Developing the Gibsonian approach to perception along evolutionary lines. Theory \& Psychology, 19, 363-389.

Withagen, R., \& Michaels, C. F. (2004). Transfer of calibration in length perception by dynamic touch. Perception \& Psychophysics, 66, 1282-1292.

Withagen, R., \& Michaels, C. F. (2005). The role of feedback information for calibration and attunement in perceiving length by dynamic touch. Journal of Experimental Psychology: Human Perception \& Performance, 31, 1379-1390.

Withagen, R., \& Michaels, C. F. (2007). Transfer of calibration between length and sweet-spot perception by dynamic touch. Ecological Psychology, 19, 1-19.

Withagen, R., \& VAN WermesKerken, M. (2009). Individual differences in learning to perceive length by dynamic touch: Evidence for variation in perceptual learning capacities. Attention, Perception, \& Psychophysics, 71, 64-75.

\section{NOTES}

1. It is important to note that $I_{1}$ of a homogeneous rod is determined by its length, mass, and diameter. However, in the case of thin rods, the contribution of diameter to the major principal moment of inertia can be neglected. Hence, the formula for $I_{1}$ that we used in the equation is an approximation for thin rods.

2. In the literature on dynamic touch, there is discussion on how to compute the mechanical variables. Turvey, Carello, and colleagues argued that the moments of inertia ought to be computed with respect to the wrist, the presumed rotation point (e.g., Fitzpatrick et al., 1994; Solomon \& Turvey, 1988). Van de Langenberg et al. (2006), on the other hand, forcefully asserted that the end of the rod ought to be taken as the reference point. In the present study, we chose to compute the mechanical variables with respect to the end of the rod. The reason was that the participants were allowed to hold the rod loosely in their hand in the wielding condition. This means that the distance between the end of the rod and the wrist was likely to vary over the trials, making it difficult to compute the mechanical variables with respect to the wrist. However, as argued elsewhere, mechanical variables differ when computed with respect to different points, rendering it difficult to compare the findings of different studies (Withagen \& Michaels, 2005, note 3). Hence, the decision about which mechanical variables to consider in our data analyses is based on research that computed the mechanical variables with respect to the end of the rod.

3. All averaging and statistical tests done on correlations in Experiments 1 and 2 used the correlations' $z$-transformations.

4. One might wonder why there is a considerable difference in correlation between the experimental group and the control group in the first feedback phase, but such a difference is to be expected. Because the correlation between perceived length and actual length is computed over all the trials in the feedback phase, learning effects during this phase result in an increase of this correlation. And learning to perceive length by dynamic touch has proven to occur rather quickly in the wielding condition (e.g., Withagen \& Michaels, 2005; see also Figure 2).

5. It is beyond the scope of this article to spell out Withagen and Chemero's (2009) argument. We refer the reader who is interested in this evolutionary analysis to their article.

6. We are grateful to Margot van Wermeskerken for introducing this distinction to us. 
APPENDIX

Geometric and Mechanical Properties

of Rods Used in Experiments 1 and 2

\begin{tabular}{lccccc}
\hline Material & Length $(\mathrm{m})$ & Diameter $(\mathrm{m})$ & $m(\mathrm{~kg})$ & $M(\mathrm{~kg} . \mathrm{m})$ & $I_{1}\left(\mathrm{~kg} . \mathrm{m}^{2}\right)$ \\
\hline \multicolumn{5}{c}{ Feedback Rod Set } \\
Aluminum & 0.70 & 0.010 & 0.148 & 0.052 & 0.024 \\
Steel & 0.50 & 0.010 & 0.298 & 0.075 & 0.025 \\
Steel & 0.70 & 0.010 & 0.418 & 0.146 & 0.068 \\
Carbon & 0.76 & 0.020 & 0.099 & 0.038 & 0.019 \\
Carbon & 0.96 & 0.020 & 0.125 & 0.060 & 0.038 \\
Carbon & 0.86 & 0.020 & 0.105 & 0.042 & 0.022 \\
Aluminum & 0.50 & 0.010 & 0.105 & 0.026 & 0.009 \\
Steel & 0.60 & 0.010 & 0.370 & 0.111 & 0.044 \\
Steel & 0.46 & 0.012 & 0.395 & 0.091 & 0.028 \\
Wood & 0.70 & 0.012 & 0.052 & 0.018 & 0.008 \\
Wood & 0.86 & 0.012 & 0.068 & 0.029 & 0.017 \\
Steel & 0.56 & 0.012 & 0.481 & 0.135 & 0.050 \\
Carbon & 0.60 & 0.020 & 0.066 & 0.020 & 0.008 \\
& & Test Rod Set & & \\
Carbon & 0.71 & 0.020 & 0.093 & 0.033 & 0.016 \\
Carbon & 0.91 & 0.020 & 0.119 & 0.054 & 0.033 \\
Carbon & 1.01 & 0.020 & 0.132 & 0.067 & 0.045 \\
Carbon & 1.11 & 0.020 & 0.145 & 0.081 & 0.060 \\
Carbon & 1.21 & 0.020 & 0.158 & 0.096 & 0.077 \\
Aluminum & 0.91 & 0.016 & 0.494 & 0.225 & 0.136 \\
Steel & 0.61 & 0.012 & 0.524 & 0.160 & 0.065 \\
Steel & 0.71 & 0.012 & 0.610 & 0.217 & 0.102 \\
Wood & 0.81 & 0.012 & 0.064 & 0.026 & 0.014 \\
Wood & 1.01 & 0.012 & 0.080 & 0.040 & 0.027 \\
Steel & 0.61 & 0.008 & 0.232 & 0.071 & 0.029 \\
Steel & 0.71 & 0.008 & 0.271 & 0.096 & 0.046 \\
Steel & 0.81 & 0.008 & 0.309 & 0.125 & 0.068 \\
\hline
\end{tabular}

(Manuscript received March 31, 2009;

revision accepted for publication July 18, 2009.) 